\title{
Acute reversible kidney injury secondary to bilateral ureteric obstruction
}

\author{
Michael Organ, MD; Richard W. Norman, MD, FRCSC \\ Department of Urology, Dalhousie University, Halifax, NS
}

\begin{abstract}
Background: Acute reversible kidney injury (ARKI) secondary to bilateral ureteric obstruction (BUO) is a common urological problem. Our goals were to describe the etiology, management and outcomes of such patients identified between 2006 and 2009 and to compare them with a similar historical study published in 1982. Methods: Chart review was performed on 49 patients with AKRI secondary to BUO. ARKI was defined as $\geq 33 \%$ decrease in serum creatinine after intervention. Those with malignant and benign causes of obstruction were identified and management and outcome data were collected.

Results: Of these 49 patients, $83 \%$ had BUO secondary to malignancy, $28 \%$ of these presenting for the first time. Prevalence of bladder cancer was increased $(p=0.04)$ and cervix trended lower ( $p=0.07$ ) compared with the earlier study; prostate cancer was unchanged $(p=0.51)$. The average survival was 239 days; $90 \%$ of patients died within a year after presenting with BUO from a malignant etiology. Compared with the 1982 group, there were trends towards a decrease in the frequency of retroperitoneal fibrosis $(p=0.08)$ and an increase in bilateral ureteric calculi $(p=0.16)$ in the benign group.

Conclusions: Patients with ARKI secondary to BUO most likely have an underlying malignancy, with almost a third of them being diagnosed for the first time. Prevalence of bladder cancer increased while cervical cancer trended lower. The cause for the former is unclear; the latter may be due to aggressive screening. Prostate cancer remained unchanged despite the widespread implementation of prostate-specific antigen testing. Patients with an underlying malignancy do poorly and those with a newly diagnosed malignancy do worst. Those with ARKI secondary to benign causes did well.
\end{abstract}

\section{Introduction}

Acute reversible kidney injury (ARKI) secondary to bilateral ureteric obstruction (BUO) is a common urological problem and the underlying etiology can be malignant or benign. Malignant obstruction is often from direct tumour compres- sion of the distal ureters, most frequently from genitourinary or colon cancers. ${ }^{1}$ Although patients with BUO typically receive urgent intervention to recoup renal function, there is limited evidence that survival and quality of life are extended by doing so.

Most publications dealing with this scenario have focused on individual causes of obstruction. In a small series of 29 patients with cervical cancer and $\mathrm{B} \cup \mathrm{O}$, some patients were salvaged with prompt urinary diversion. ${ }^{2}$ A larger review looking at prostate cancer suggested there was no consensus on how to manage BUO in advanced disease. ${ }^{3}$ Individual case series on breast and esophageal cancer suggested ureteric diversion reversed the kidney injury and allowed further treatment of the systemic disease., ${ }^{4,5}$ Despite the controversy in the management of this condition and the accepted poor prognosis, most patients receive percutaneous nephrostomy tubes (PNTs), retrograde ureteric stents or both. ${ }^{1,6-8}$

In contrast, those with benign causes have cause-specific interventions and do well. We sought to describe the etiology, management and outcome in a contemporary cohort of patients with ARKI secondary to BUO who had malignant or benign disease and to compare them to a similar historical group. ${ }^{9}$

\section{Methods}

Chart review was performed on patients with ARKI secondary to BUO admitted to the Capital District Health Authority in Halifax, Nova Scotia, Canada, between 2006 and 2009 (referred to in this paper as the 2009 group). ARKI was defined by $\geq 33 \%$ reduction in serum creatinine after intervention. Those with bladder outlet obstruction $(n=7)$ or who refused intervention $(n=1)$ were excluded.

Once the cause of the obstruction was determined, patients were classified into malignant or benign groups. Independent-sample t-tests were used to compare serum creatinine $(\mu \mathrm{mol} / \mathrm{l})$ in the malignant and benign groups (creatinine \pm standard deviation.) Each patient was labeled 
with specific cancer or underlying diagnosis depending upon whether the etiology was malignant or benign. The frequency of individual causes was compared to a historical study $^{10}$ (referred to in this paper as the 1982 group.) using either Pearson's chi-squared or Fisher's exact tests. KaplanMeyer time to event survival analysis was calculated with SPSS (SAS Institute, Cary, NC). Survival is expressed as mean survival (days), with a $95 \%$ confidence interval.

Statistical comparison was not possible in those instances when raw data from the 1982 group were unavailable.

Ethical approval was granted by Capital District Health Authority Research Services.

\section{Results}

The 2009 group included 49 patients identified with AKRI secondary to BUO confirmed by ultrasonography (US) and/ or computerized tomography (CT); in 1982, most (48/50) patients were diagnosed with cystoscopy and retrograde pyelography.

Forty $(82 \%)$ had a malignant cause for their obstruction with an average age of 73 years at presentation (compared with 60 years in the 1982 group) while $9(18 \%)$ had a benign cause with an average age of 59 years. Causes of obstruction were tallied (Table 1).

Comparing the two groups, the 2009 group showed an increase in the prevalence of bladder cancer $(p=0.04)$, a trend towards a decrease in cervical cancer $(p=0.07)$ and no change in the frequency of prostate cancer $(p=0.51)$ (Table 1). In the 2009 group, there were 13 females and 27 males with a malignant cause of obstruction; females presented most commonly with cervical or bladder cancer, while male patients presented most often with prostate or bladder cancer (Fig. 1, Fig. 2).

In the 2009 group, patients with ARKI secondary to malignant BUO had a mean survival of 239 days (range: 145-334) (Fig. 3); median was 133 days. Patients with a pre-existing malignancy survived longer with an average of 292 days (range: 168-497) days while those with a newly diagnosed

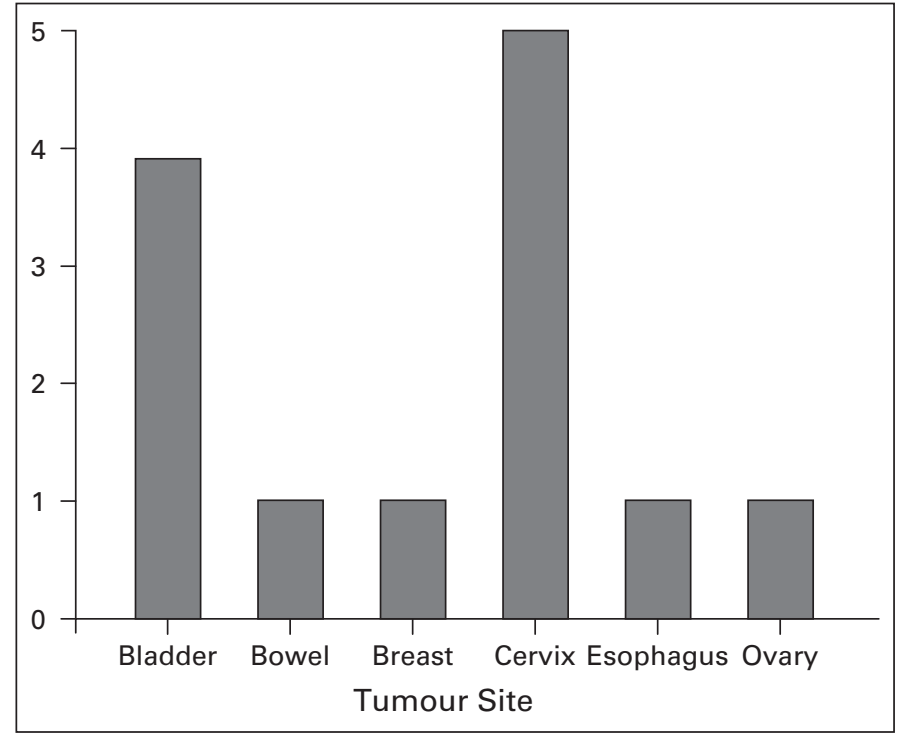

Fig. 1. Distribution of females presenting with malignant obstruction in 2009 group $(n=13)$.

malignancy survived only 109 days (range: 49-168) days $(p=0.04)$ (Fig. 4). There was no difference in the survival of men compared to women with malignancy with men surviving 195 (range: 116-274) and women surviving 331 (range: 145-334) days ( $p=0.28$ ) (Fig. 5). When comparing the survival of patients with different kinds of cancer there was no statistically significant difference between the two groups (Table 2).

In the 1982 group, $75 \%$ of patients who had nephrostomy tubes had them done with an open procedure, whereas in the contemporary group all were done percutaneously (Table 3). In the 2009 group who had obstruction from a benign etiology, $56 \%$ had bilateral ureteric calculi and $22 \%$ had retroperitoneal fibrosis. All of the patients with calculi initially received bilateral ureteric stents. One patient with retroperitoneal fibrosis had a PNT inserted and the other had bilateral ureteric stents. Compared with the 1982 group, there were trends towards a decrease in the frequency of

\begin{tabular}{|c|c|c|c|c|c|c|c|}
\hline \multirow[b]{2}{*}{ Site } & \multicolumn{2}{|c|}{ Malignant } & \multirow[t]{2}{*}{$p$} & \multirow[b]{2}{*}{ Etiology } & \multicolumn{2}{|c|}{ Benign } & \multirow[t]{2}{*}{$p$} \\
\hline & $\begin{array}{l}1982 \\
n=32\end{array}$ & $\begin{array}{l}2009 \\
n=40\end{array}$ & & & $\begin{array}{l}1982 \\
n=12\end{array}$ & $\begin{array}{c}2009 \\
n=9\end{array}$ & \\
\hline Cervix & 11 (29\%) & $5(13 \%)$ & 0.07 & $\begin{array}{l}\text { Retroperitoneal } \\
\text { fibrosis }\end{array}$ & $8(67 \%)$ & $2(22 \%)$ & 0.08 \\
\hline Prostate & $8(21)$ & $11(28)$ & 0.51 & Ureteric stones & $2(17)$ & $5(56)$ & 0.16 \\
\hline Bladder & $5(13)$ & $13(33)$ & 0.04 & Ligated ureters & $2(17)$ & 0 & - \\
\hline Colon & $5(13)$ & $5(13)$ & 1.0 & Other & 0 & $2(22)$ & - \\
\hline Ovary & $5(13)$ & $1(3)$ & 0.10 & & & & \\
\hline Other & $3(8)$ & $2(5)$ & - & & & & \\
\hline Lymphoma & $1(3)$ & $3(8)$ & 0.62 & & & & \\
\hline
\end{tabular}




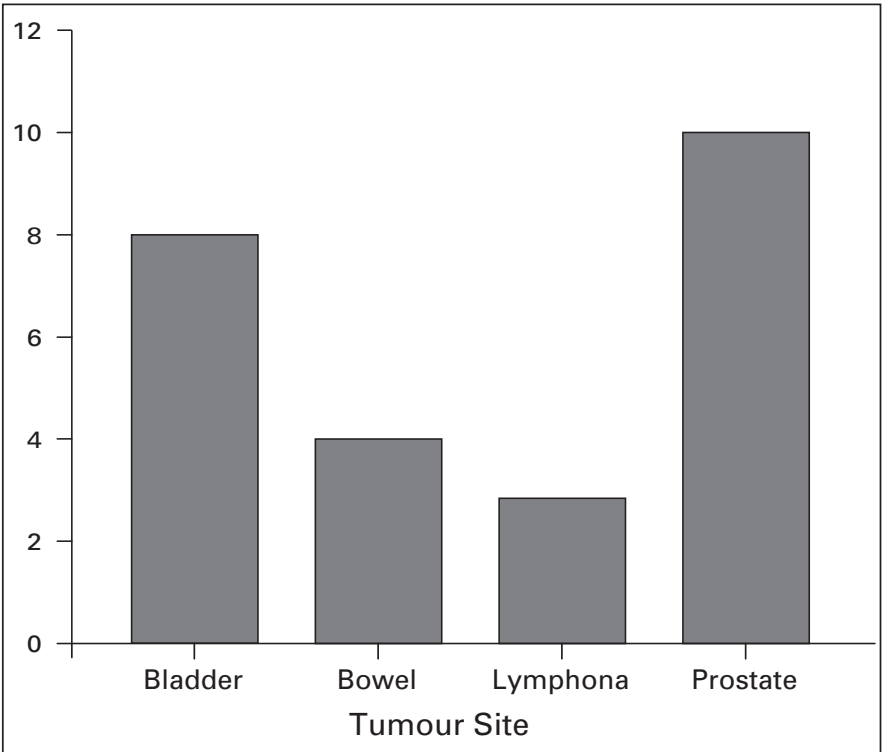

Fig. 2. Distribution of males presenting with malignant obstruction in 2006 to 2009 by tumour site $(n=27)$.

retroperitoneal fibrosis $(p=0.08)$ and an increase in bilateral ureteric calculi ( $p=0.16$ ) (Fig. 6).

Presenting serum creatinine in the malignant and benign groups was the same on admission $(733 \pm 378,721 \pm 486$ $(p=0.94)$, respectively) and at discharge $(222 \pm 129$, $205 \pm 120$ ( $p=0.72)$, respectively). None of this 2009 cohort required dialysis.

\section{Discussion}

ARKI has been defined in multiple studies using varying changes in serum creatinine, urine output, need for renal replacement therapy and estimated glomerular fil-

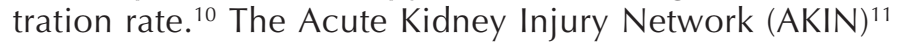
has proposed a definition of AKI based on the Risk, Injury, Failure, Loss, and End-stage Kidney (RIFLE) classification. ${ }^{12}$ Diagnostic and classification criteria are based on changes in serum creatinine from baseline values. Strict adherence

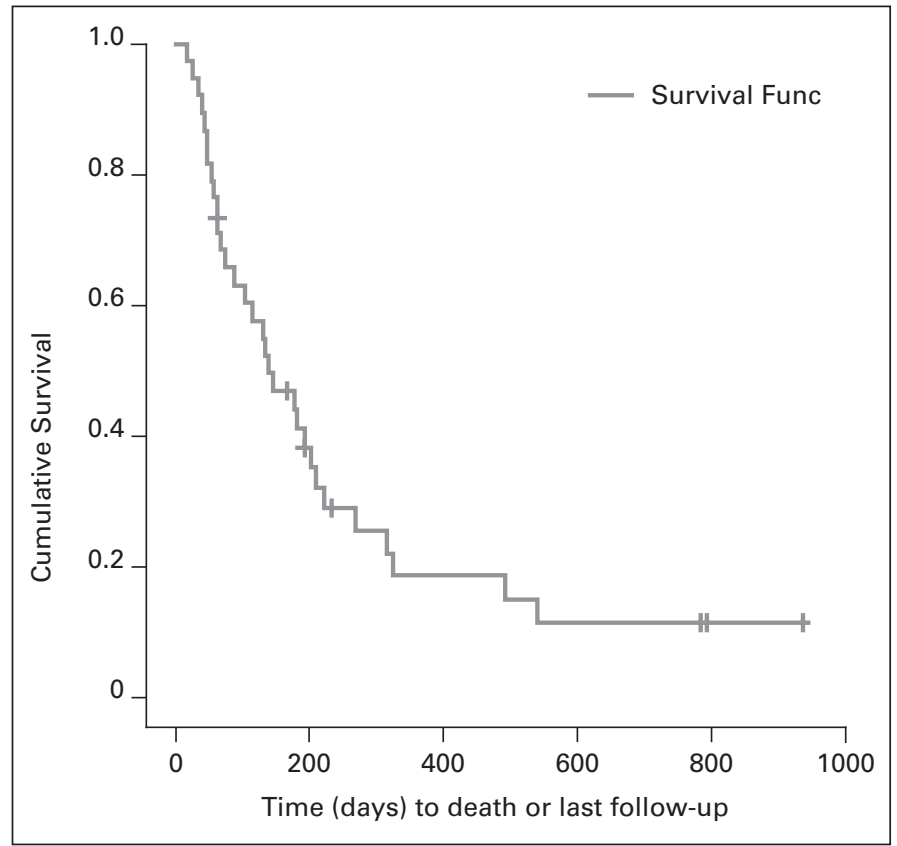

Fig. 3. Overall survival of the 2006-2009 group with malignant causes of bilateral ureteric obstruction.

to these criteria was not possible in our in-hospital study because neither baseline nor final serum creatinine values were available - values were already elevated at the time of admission and the ultimate nadir was unknown at time of discharge.

Recognizing that there are ongoing controversies regarding standardized definitions of ARKI and recovery, ${ }^{12-14}$ we used $\geq 33 \%$ decrease in serum creatinine after intervention as confirmation of AKRI following consultation with our local nephrologists.

Most patients who presented with ARKI secondary to BUO had an underlying malignancy and most of these were genitourinary (bladder, cervix or prostate). This is consistent with the existing literature and similar to a 1982 study at our institution. ${ }^{9,15}$ In both the benign and malignant groups, the degree of renal impairment as measured by presenting serum creatinine was similar and did not serve as a differentiating variable. After relief of obstruction with percutaneous nephrostomy tubes or ureteric stents, renal function improved significantly and was decreasing on discharge in both groups. Multiple studies have shown both stents and nephrostomy tubes are effective methods to restore kidney function after ureteric obstruction. ${ }^{2,3,10,16-19}$ Advancements in and availabil- 


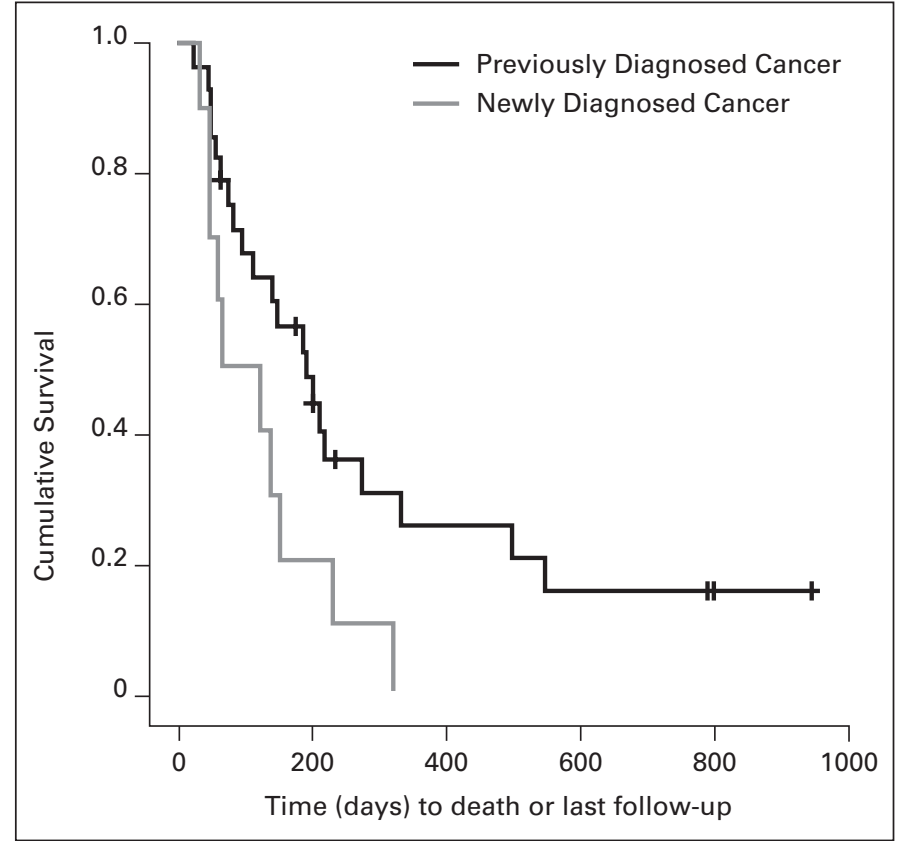

Fig. 4. Survival of patients with bilateral ureteric obstruction from a newly diagnosed malignancy compared with those who had a known malignancy (2006-2009).

ity of imaging techniques have led to less invasive diagnosis and treatment of patients with BUO in the 2009 group.

There was a trend toward a decreasing percentage in the number of patients presenting with BUO secondary to cervical cancer compared with 1982. It is possible that aggressive screening for cervical cancer over the past 25 years contributed to this observation. ${ }^{20}$ On the other hand, we did not see the expected reduction in prostate cancer patients with BUO despite the widespread availability of PSA screening over the past few decades. It is unknown why more patients are currently presenting with bladder cancer as the cause of obstruction.

Patients with malignancy had poor outcomes and most died within a year. Our mean survival of 239 days is similar to previous rates described in the literature. One study

\begin{tabular}{lccc}
\hline Table 3. Interventions used in the 2009 malignant group \\
\hline Tumour site & $\begin{array}{c}\text { Percutaneous } \\
\text { nephrostomy } \\
\text { tubes }\end{array}$ & $\begin{array}{c}\text { Retrograde } \\
\text { ureteric stents }\end{array}$ & Both \\
\hline Bladder & 8 & 2 & 2 \\
Bowel & 1 & 2 & 2 \\
Breast & - & - & 1 \\
Cervix & 1 & 3 & 1 \\
Lymphoma & - & 3 & - \\
Ovary & 1 & - & - \\
Prostate & 7 & 3 & - \\
\hline
\end{tabular}

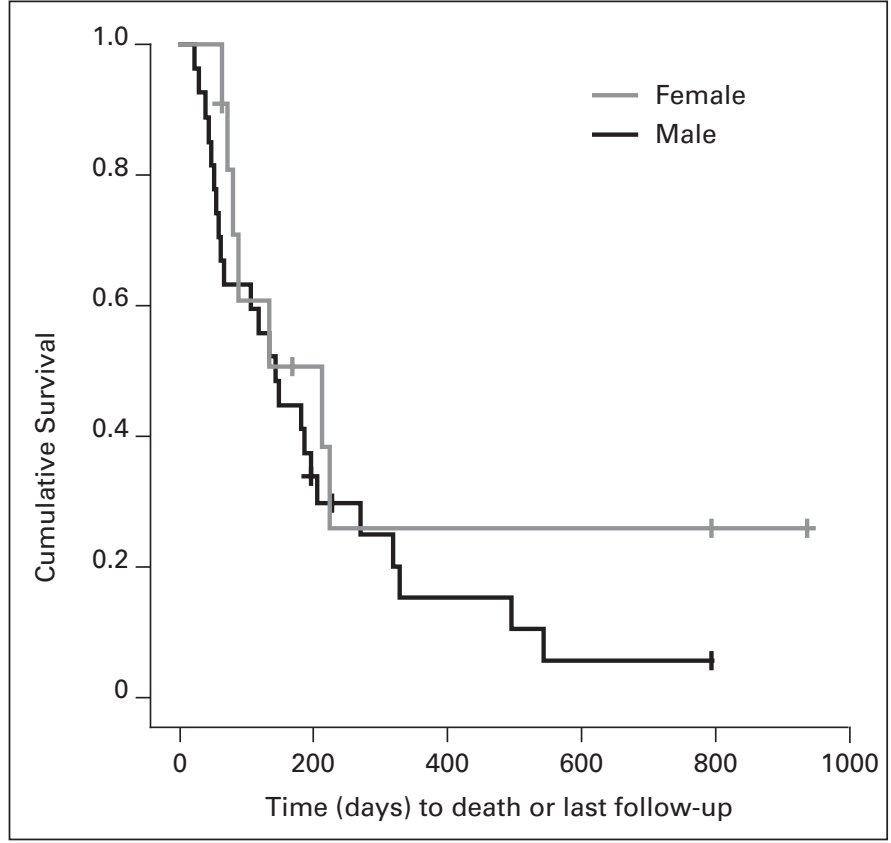

Fig. 5. Comparison of survival of men with malignant bilateral ureteric obstruction to women (2006-2009).

described survival post-obstruction from a genitourinary malignancy to be only 5 to 12 months. ${ }^{16}$ In another, patients with prostate cancer treated with PNTs averaged 7.5 months until death. ${ }^{18}$ In a large series with 125 patients with malignancy, the average survival was 227 days post-obstruction. ${ }^{19}$ Patients with prostate cancer had no change in their survival with a mean of 200 days in the 2009 group compared with a median survival of 190 days in 1982 group. Interestingly, patients with colon cancer had the shortest mean survival of 162 days in the 2009 group compared with in 1982 (median survival of 22 days). It is difficult to compare mean survival rates to median survival in a previous study, but the similarities in results suggest that limited progress has been made in improving the poor prognosis of these patients.

The encouraging exception in the 2009 group was cervical cancer where survival seemed to be better than other malignancies, with 3 patients alive 1 to 3 years post-obstruction. This should be interpreted cautiously as the numbers were small with regards to individual cancers and the results were difficult to generalize. Patients with cervical cancer also had the longest median survival of 277 days in the 1982 group. $^{9}$

The reason that patients with a pre-existing diagnosis of cancer survived longer than those with a newly diagnosed malignancy is unclear. It may be because those patients who present with bilateral obstruction as their initial symptom of malignancy have more aggressive tumours. 


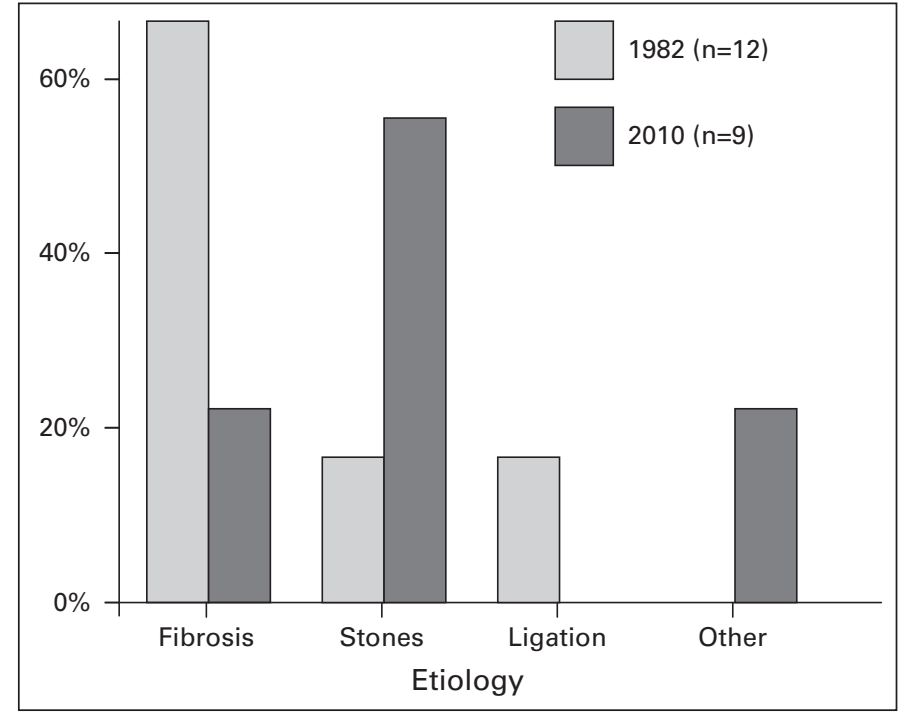

Fig. 6. Causes of benign obstruction (1982 compared with 2009).

\section{Conclusions}

Based on our observations, patients with ARKI secondary to malignant BUO should be counselled that their prognosis is poor. Bypassing the obstruction has been shown to be a successful way to prolong life, ${ }^{1,2,17}$ but most will not survive past a year despite receiving adequate intervention for their ureteric obstruction. The quality of life after intervention is not well-described, but is likely poor. No patients were reviewed who chose only a palliative approach to treat their acute obstruction, but this may be an appropriate option to consider.

With respect to the patients with a benign cause of their obstruction, there were trends toward a decreasing number of patients who presented with retroperitoneal fibrosis and an increasing number presenting with bilateral ureteric calculi.

Future studies might consider reviewing patients presenting with $\mathrm{BUO}$ and non-reversible $\mathrm{AKI}$, as well as those with unilateral ureteric obstruction in a solitary kidney with reversible and non-reversible AKI.

Competing interests: None declared.

This paper has been peer-reviewed.

\section{References}

1. Allen DJ, Longhorn SE, Philp T, et al. Percutaneous urinary drainage and ureteric stenting in malignant disease. Clin Oncol (R Coll Radiol) 2010;22:733-9.

2. Lee SK, Jones HW 3rd. Prognostic significance of ureteral obstruction in primary cervical cancer. Int J Gynaecol Obstet 1994;44:59-65.

3. Rollig C, Wockel A, Weissbach L. Management of obstructive uropathy patients with advanced prostate cancer - a systematic review. Onkologie 2009;32:680-4.

4. Akmal M, Kaptein EM, Bertram J, et al. Acute renal failure due to bilateral ureteral obstruction by metastases from breast cancer. Nephron 1986;42:23-8.

5. Marlin ES, Hyams ES, Dulabon L, et al. Metastatic esophageal adenocarcinoma to the prostate presenting with bilateral ureteral obstruction. Can I Urol 2010;17:5035-7.

6. Chung SY, Stein RJ, Landsittel D, et al. 15-year experience with the management of extrinsic ureteral obstruction with indwelling ureteral stents. J Urol 2004;172:592-5.

7. Ishioka J, Kageyama Y, Inoue $M$, et al. Prognostic model for predicting survival after palliative urinary diversion for ureteral obstruction: Analysis of 140 cases. J Urol 2008;180:618-21; discussion 621.

8. Gasparini $M$, Carroll P, Stoller M. Palliative percutaneous and endoscopic urinary diversion for malignant ureteral obstruction. Urology 1991;38:408-12.

9. Norman RW, Mack FG, Awad SA, et al. Acute renal failure secondary to bilateral ureteric obstruction: Review of 50 cases. Can Med Assoc J 1982; 127:601-4.

10. James M, Pannu N. Methodological considerations for observational studies of acute kidney injury using existing data sources. J Nephrol 2009;22:295-305.

11. Acute Kidney Injury Network. www.AkINet.org. Accessed November 10, 2011,

12. Mehta R, Kellum J, Shah S, et al; AKINetwork. Acute Kidney Injury Network: Report of an Initiative to Improve Outcomes in Acute Kidney Injury Critical Care 2007;11:R31. doi:10.1186/cc5713. http:// ccforum.com/content/11/2/R31 (Accessed November 8, 2011).

13. Martin RK. Acute kidney injury: advances in definition, pathophysiology, and diagnosis. AACN Crit Care 2010;21:350-6.

14. Macedo E, Bouchard J, Mehta RL. Renal recovery following acute kidney injury. Curr Opin Crit Care 2008; 14:660-5.

15. Higa I, Imagawa A. Clinical studies on 61 patients with post-renal acute renal failure caused by ureteral obstruction. Hinyokika Kiyo 1987;33:1005-10.

16. Barton DP, Morse SS, Fiorica JV, et al. Percutaneous nephrostomy and ureteral stenting in gynecologic malignancies. Obstet Gynecol 1992;80:805-11.

17. Hubner W, Leodolter S, Nemecek B, et al. Hydronephrosis as a late complication in gynecologic neoplasms: Current possibilities for sensible urinary drainage. Geburtshilfe Fravenheilkd 1988;48:255-9.

18. Nariculam J, Murphy DG, Jenner C, et al. Nephrostomy insertion for patients with bilateral ureteric obstruction caused by prostate cancer. Br J Radiol 2009;82:571-6.

19. Pappas P, Stravodimos KG, Mitropoulos D, et al. Role of percutaneous urinary diversion in malignant and benign obstructive uropathy. J Endourol 2000;14:401-5.

20. Cannistra SA, Niloff JM. Cancer of the uterine cervix. N Engl J Med 1996;334:1030-8.

Correspondence: Dr. Richard W. Norman, Suite 620, 5991 Spring Garden Rd., Halifax, NS B3H 1Y6; richard.norman@dal.ca 